\title{
Proposta de um método multicritério para escolha múltipla
}

\author{
Henrique Rego Monteiro da Hora ${ }^{\mathrm{a} *}$, Helder Gomes Costa ${ }^{\mathrm{b}}$ \\ a*henrique.dahora@iff.edu.br, IFF, Brasil \\ bhgc@vm.uff.br, UFF, Brasil
}

\begin{abstract}
Resumo
Este trabalho tem como objetivo propor uma solução multicritério para a problemática de escolha múltipla dentro do mesmo problema decisório, partindo da hipótese de que as $n$ melhores alternativas em uma ordenação não são necessariamente o melhor conjunto de $n$ alternativas. Por meio de uma pesquisa experimental, simula-se um problema de recrutamento, onde se deseja escolher três candidatos entre dez. O MEM - Multiplex Electionis Methodus - oferece uma solução para esse problema partindo da modelagem tradicional dos problemas decisórios, com matriz de pagamentos e vetor de pesos, e agregando uma matriz de complementaridade entre critérios. Os resultados apontam que a melhor opção tripla não são as três opções melhores colocadas em uma ordenação simples e cumpre-se o objetivo do trabalho ao confirmar a hipótese declarada, contrariando a decisão intuitiva de admitir que as $n$ primeiras alternativas são o melhor conjunto de $n$ alternativas.
\end{abstract}

\section{Palavras-chave}

Decisão múltipla. Método multicritério. Teoria da decisão.

\section{Introdução}

Um modelo decisório aspira por representar a realidade de modo racional, lógico, coerente e intencional (Rogers et al., 2000, p. 189). Em sua dimensão mais básica, um processo de tomada de decisão pode conceber-se como a eleição por parte de um centro decisor (um indivíduo ou um grupo de indivíduos) de "o melhor" entre "os possíveis" (Romero, 1996, p. 14). Os problemas analíticos surgem na hora de definir "o melhor" e "o possível" em um determinado contexto decisional onde as decisões envolvem uma série de objetivos (critérios) conflitantes (Ehrgott, 2005, p. 4) e são subjetivas (Abramczuk, 2009, p. 4).

Várias ferramentas de análise de decisões são desenvolvidas no processo de tomada de decisão, cada uma com seus pontos fortes e fracos. Cabe ao tomador de decisão (decisor) compreender o contexto, reconhecer as vantagens e desvantagens dessas ferramentas e sua adequação ao processo de tomada de decisão (Bhushan \& Rai, 2004, p. 6).

Belton \& Stewart (2002, p. 15) caracterizam a problemática de escolha de um conjunto de alternativas como sendo um problema de seleção de portfólio. Nesse contexto, várias abordagens têm sido desenvolvidas dentre as quais a clássica proposta de Markowitz (1952, p. 77). No entanto, conforme destacado em Almeida \& Duarte (2011, p. 302), poucas consideram a presença de sinergia.

Por outro lado, a complexidade das situações de decisão demanda que se considere o uso de múltiplos critérios, destacando-se nesse contexto as modelagens baseadas nos métodos originados do ELECTRE (Rogers et al., 2000) ou do AHP (Saaty, 1991). Também nesse contexto, a maioria das modelagens não considera explicitamente a presença de sinergias, embora Almeida \& Duarte (2011, p. 302) e o método ANP (Saaty \& Vargas, 2006) considerem a presença dessas sinergias.

Pode-se dizer que, em geral e especialmente no campo das engenharias, não existe uma superioridade de um método em relação a outro. 0 estudo cuidadoso da natureza do problema a analisar conduzirá à escolha do método multicritério mais adequado (Romero, 1996, p. 94). Por fim, a escolha de qual método utilizar 
depende das características do problema enfrentado (Goicoechea et al., 1982, p. 167).

A presente proposta se diferencia das demais ao considerar, de forma inédita, a problemática da escolha múltipla em presença de sinergias, admitindo como mensurável a complementaridade entre critérios, com um número conhecido de alternativas a serem escolhidas.

Vincke (1992, p. 3) apresenta duas situações hipotéticas que ilustram a problemática de decisões múltiplas: a primeira versa sobre a localização de duas usinas hidroelétricas entre oito localizações possíveis e aponta a necessidade de combinar e avaliar combinatoriamente as opções tomadas dois a dois, pois a atuação de uma influencia diretamente a outra; e a segunda compreende a escolha de dois candidatos entre 20, para duas vagas em um banco, no qual, se forem trabalhar isoladamente, em posições semelhantes, o problema deve ser encarado como duas escolhas simples, mas se forem trabalhar como uma equipe, o problema deve ser abordado considerando as alternativas conjuntamente, dado que problemas de otimização combinatória têm um conjunto finito de soluções viáveis (Ehrgott, 2005, p. 198).

Essa problemática de escolha múltipla é uma complementação da definição da problemática P. $\alpha$ de escolha (Figueira et al., 2005, p. 143). Há na literatura científica a proposição de uma derivação dessa problemática (Belton \& Stewart, 2002, p. 15), que é a problemática de portfólio, a qual consiste em escolher um subconjunto de alternativas, dentro de um conjunto maior de alternativas, considerando não somente as características individuais das alternativas, mas também o modo em que elas interagem, apresentando sinergias positivas ou negativas.

0 problema (questão) desta pesquisa pode ser explicitado de forma sucinta e precisa como: "Em um problema de decisão em que se deseja escolher mais de uma opção, os $\mathrm{n}$ primeiros elementos de uma lista ordenada compõem a melhor decisão?", e, como hipótese à resposta desse problema, formula-se: "Os n primeiros elementos de uma ordenação não compõem, necessariamente, a melhor escolha em conjunto. É necessário considerar a complementaridade intracritérios para definir o(s) melhor(es) grupo(s)".

Considerando que não existe um melhor método, e o mais apropriado para a situação (Rogers et al., 2000 , p. 84), e que diferentes problemas de decisão requerem diferentes abordagens para solucioná-los (Almeida-Dias et al., 2010, p. 565), este trabalho tem como objetivo principal propor um método que contemple a escolha múltipla em problemas multicritério, considerando a sinergia intracritérios, a partir de uma matriz de pagamentos tradicional, quando o problema decisório concernir na escolha de um subconjunto com número de elementos predeterminados em um conjunto de alternativas.

\section{Revisão bibliográfica}

\subsection{Independência e interação entre critérios}

$\mathrm{Na}$ abordagem prescritiva, onde o problema decisório é estruturado, o item mais delicado no preparo racional para a ação é, sem dúvida, no conceito tanto de psicólogos quanto economistas, a conversão de objetivos em critérios (Kaufmann, 1975, p. 35). Essa questão tem sua capacidade agravada ao considerar-se a interação entre critérios (Marichal \& Roubens, 2000, p. 642).

É sabido que há critérios conflitantes em problemas decisórios (Gomes et al., 2002, p. 63), uma vez que diferentes critérios representam diferentes dimensões das alternativas, eles podem entrar em conflito. Por exemplo, um critério "custo" pode conflitar com um critério "lucro". Em geral, esses conflitos não são tratados (Triantaphyllou, 2000, p. 2). Assumindo que nem sempre dois critérios apontam para a mesma decisão (Hansson, 2005, p. 62), surge a necessidade de uma abordagem que aglomere, considere e pondere todos os critérios e trate os casos conflitantes, para não viciar o modelo decisório.

Muitos problemas de decisão não podem ser estruturados como hierarquia, pois envolvem interação e dependência entre as partes (Saaty \& Vargas, 2006, p. 7). As decisões alcançadas por estruturas que não consideram a complementaridade entre os elementos da decisão podem ser significantemente diferentes das que a consideram.

Enlaces de realimentação são comuns quando a estruturação hierárquica do problema decisório se desfaz. Isso ocorre quando um conceito meio influencia um conceito fim (Gomes et al., 2002, p. 55; Gomes \& Gomes, 2012, p. 52). 0 mesmo pode ser afirmado para influência intercritérios.

É recomendado que a definição do conjunto de critérios seja feita de modo que eles sejam bem definidos, distintos uns dos outros (Kaufmann, 1975, p. 35) e mutuamente exclusivos, ou seja, não pode haver dúvida da alocação de um determinado resultado (Rogers et al., 2000, p. 7; Santos, 2011, p. 198). Claramente, a definição de critério implica que cada um é preferencialmente independente no conjunto de critérios (Vincke, 1992, p. 37, 113).

Corroborando com essas proposições, também é recomendado que haja independência entre critérios (Gomes et al., 2002, p. 114-115; Gomes \& Gomes, 
2012, p. 119-120), sendo essa independência definida em três níveis:

- Isolabilidade de cada critério: em que os julgamentos de $g_{k}(a)$ e $g_{k}(b)$ devem ser feitos considerando-se unica e respectivamente $g_{k}(a)$ e $g_{k}(b)$;

- Separabilidade de cada subfamília: independência no sentido de preferência, em que separando-se o conjunto de critérios em dois subconjuntos complementares, é possível se chegar ao mesmo resultado;

- Independência de ordem estrutural: definida como ausência de fatores que influenciam conjuntamente vários critérios. Em outras palavras, num conjunto $\mathrm{F}$ de critérios, dois ou mais elementos desse conjunto formam um subconjunto $\mathrm{J}$ tal que os critérios de $\mathrm{J}$ apresentam uma ligação suscetível de influência conjunta entre as performances dos critérios, ocasionando certa redundância.

Raramente o desempenho de uma ação a, segundo um critério j, $g_{j}(a)$, é proveniente do conhecimento de uma única característica. Na verdade, esse julgamento constitui na maioria dos casos uma síntese de informações mais ou menos complexas (Gomes et al., 2002, p. 114; Gomes \& Gomes, 2012, p. 119).

Os critérios (e alternativas) podem estar interligados, de forma que determinado critério possa refletir parcialmente outro (Gomes, 2007, p. 24), e ao invés de simplificar a estrutura, ignorando essa relação, um modelo racional tenta avaliar as influências quantitativamente (Tietz, 1992, p. 303). A importância geral de um critério não é somente determinada pelo seu valor, mas sim por todo o conjunto de critérios que interagem entre si (Marichal \& Roubens, 2000, p. 643).

Já é identificada em problemas multicritério a interação entre os critérios e a necessidade em tratar essa situação com a criação de uma matriz 1, chamada Matriz de interação entre critérios, que armazena valores para correção dos pesos dos critérios nas funções de agregação, agindo de modo compensatório (Marichal \& Roubens, 2000, p. 643-645).

Para os métodos de sobreclassificação da família ELECTRE, é proposto um índice $\mathrm{k}$ incorporado ao cálculo da matriz de concordância para compensação das situações de interação entre critérios (Figueira et al., 2009, p. 482). De modo análogo, modelagem semelhante é proposta para a família PROMETHEE nas funções de agregação por meio de um índice a (Corrente et al., 2012, p. 473).

\subsection{Métodos semelhantes}

0 primeiro método desenvolvido pela Escola Francesa, inicialmente chamado somente de ELECTRE e, após o desenvolvimento dos demais, denominado ELECTRE 1 (e suas versões lv e ls), utiliza as "relações de sobreclassificação (outranking) de tal modo que seja obtido um subconjunto k de alternativas possíveis, também chamado de mínimo subconjunto dominante" (Gomes et al., 2002, p. 202-203), apesar de ter sido desenvolvido originalmente para a escolha de uma única alternativa no subconjunto dominante, em um grafo acíclico (Roy \& Vanderpooten, 1996, p. 23).

Na família PROMETHEE há a proposição do método PROMETHEE V, que após uma ordenação comandada pelo método PROMETHEE 11, utiliza a programação linear inteira binária para selecionar um subconjunto de alternativas que maximiza um indice de sobreclassificação, levando em consideração restrições conhecidas a priori (Gomes et al., 2004, p. 135).

Almeida \& Duarte $(2011$, p. 301, 304) propõem uma modelagem multicritério de problemas de seleção de portfólio que procura mensurar a sinergia entre critérios e propõem uma função objetivo via programação não linear 0-1 que procura maximizar um valor do portfólio e o valor de sinergia entre critérios.

Em todos os casos de seleção de subconjunto (ELECTRE 1; PROMETHEE V e Almeida \& Duarte, 2011), o tamanho desse subconjunto não é conhecido ou determinado a priori e só será conhecido após os procedimentos terem sido realizados e as alternativas, identificadas.

\section{Abordagem metodológica}

\subsection{Procedimentos metodológicos}

A pesquisa bibliográfica reportada, além de fornecer o arcabouço técnico-científico e base conceitual do artigo, fornece subsídio para fundamentar a estrutura lógica e funções de agregação do método proposto, perfazendo assim, além da discussão entre autores, a justificativa técnica de posições adotadas na elaboração dos mecanismos internos da proposição.

Os procedimentos internos do método proposto foram implementados com o auxílio do software MatLab R2012a, versão 7.14.0.739, para Windows 64bits. Para confecção dos gráficos foram utilizados os recursos de planilha eletrônica.

Para ilustração do método proposto, simula-se uma situação de escolha dentro da problemática adequada ao que o método se propõe, com dados reais, realizando uma análise de sensibilidade do tau $(\tau)$ de corte da escolha.

Para verificação da estabilidade do resultado é realizada uma análise de sensibilidade por meio da correlação rho de Spearman, para comparar 
os diferentes resultados obtidos pela variação dos parâmetros do problema. Essa técnica é utilizada, por exemplo, por Brans et al. (1986, p. 237) para comparação entre as ordenações obtidas pelo PROMETHEE e ELECTRE 111.

A correlação de Spearman é utilizada para medir a relação entre duas variáveis de uma escala ordinal (Gravetter \& Wallnau, 2008, p. 541). Utiliza-se neste trabalho o software SPSS 13 para o cálculo coeficiente rho.

0 coeficiente de correlação de postos de Spearman, denotado por $r_{s}$, é simplesmente o rho de Pearson calculado para os postos dos valores de $x$ e $y$ (Equação 1), portanto:

Equação 1. Coeficiente de correlação rho de Spearman (Pagano \& Gauvreau, 2004, p. 358).

$$
r_{s}=\frac{\sum_{i=1}^{n}\left(x_{r i}-\bar{x}_{r}\right) \cdot\left(y_{r i}-\bar{y}_{r}\right)}{\sqrt{\left[\sum_{i=1}^{n}\left(x_{r i}-\bar{x}_{r}\right)^{2}\right] \cdot\left[\sum_{i=1}^{n}\left(y_{r i}-\bar{y}_{r}\right)^{2}\right]}}
$$

onde $x_{r i}$ e $y_{r i}$ são os postos associados com o $i$-ésimo indivíduo em vez das observações reais.

Os resultados alcançados pelo MEM (Multiplex Electionis Methodus, em latim) são analisados de três formas.

- Comparando-se o resultado da uma ordenação resultante da média aritmética ponderada da matriz de pagamentos e o vetor de pesos, tomando as três primeiras alternativas, com o resultado do método proposto;

- Apresentando-se a solução por meio de um gráfico de área, onde os valores foram plotados junto com uma série que representa o tau $(\tau)$ de corte, que ilustra a abrangência de uma decisão graficamente;

- Analisando-se a frequência das alternativas nas primeiras dez posições do método proposto, a fim de verificar qual é mais propensa à complementaridade.

\section{MEM: Multiplex Electionis Methodus}

\subsection{Apresentação}

Na resolução de problemas multicritério, as inspirações para sua solução podem advir por meio de uma matemática não mais sofisticada do que a álgebra do ensino médio, em detrimento do uso de matemática avançada. A verdade é que quanto mais simples a ferramenta analítica, melhor (Raiffa, 2002, p. 176). 0 método brasileiro TODIM, por exemplo, teve seu desenvolvimento norteado para se obter uma ferramenta de tomada de decisão que pudesse ser utilizada por qualquer um que tivesse conhecimento básico de álgebra linear (Gomes \& Lima,
1991, p. 117). Corroborando com isso, a simplicidade e a interatividade devem ser as linhas de força da atividade de apoio à decisão (Bana \& Costa, 1993, p. 12). Vargas (1990, p. 2) atribui ser o sucesso do AHP consequência da sua simplicidade e robustez.

Nesse sentido, o MEM apresenta mecanismos internos que privilegiam o bom desempenho médio da coletividade, contra excelentes desempenhos individuais agregados a desempenhos não tão bons.

0 campo multicriterial é vasto, possuindo diversos métodos, e todos têm em comum o foco de apoiar o decisor para a escolha da melhor (ou melhores) alternativa(s) em determinados problemas considerados não tratáveis pelos procedimentos intuitivos. As fases para utilização do método proposto são divididas em cinco passos:

- Elaborar a matriz $\mathrm{A}_{\mathrm{i}, \mathrm{j}}$ de pagamentos das i alternativas à luz dos j critérios;

- Elaborar a matriz $\mathrm{C}_{\mathrm{j} \mathrm{j}^{\prime}}$ de critérios complementares, registrando o relacionamento interno entre os critérios;

- Elaborar uma matriz linha (vetor) $\mathrm{W}_{\mathrm{j}}$, contendo o peso de cada critério, para definição do perfil da escolha;

- Estabelecer o valor do tau $(\tau)$ de corte;

- Calcular a matriz $\mathrm{B}_{1, \mathrm{~m}}$, que agrega as avaliações das matrizes $\mathrm{A}, \mathrm{C}$ e $\mathrm{W}$, combinando as alternativas $\mathrm{n}$ a $\mathrm{n}$, levando em consideração valor do tau $(\tau)$ corte.

\subsection{Elaboração da matriz de pagamento}

$A_{i, j}$ vem a ser uma matriz comum de pagamentos, onde $\mathrm{a}_{\mathrm{ij}}$ contém a avaliação $\mathrm{g}$ da alternativa i à luz do critério $\mathrm{j}$, mostrando o desempenho de cada alternativa diante dos critérios, considerando o sentido de preferência.

A avaliação das alternativas, ou medição, é mais facilmente visualizada como o processo de ligar conceitos abstratos a indicadores empíricos, incorporando elementos teóricos e empíricos a esse processo (Carmines \& Zeller, 1979, p. 10).

\subsection{Definição da matriz de complementaridade entre critérios}

A matriz $C_{j, j}$, matriz quadrada de ordem $\mathrm{j}$, contém o grau de complementaridade de um critério j sobre outro critério j, ou seja, relaciona os critérios segundo uma escala que procura medir essa complementaridade. Em outras palavras, quantificar suas influências (Tietz, 1992, p. 303). Essa mensuração da complementaridade é avaliada paritariamente, dentro do conjunto dos critérios. Almeida \& Duarte (2011, p. 303) afirmam 
que termo sinergia é repleto de subjetividade, mas em sua essência traz a noção de cooperação entre as partes envolvidas.

0 algoritmo proposto permite que o relacionamento seja reflexivo ou não, isto é, que a influência de um dado critério j em outro dado critério j' seja em uma determinada intensidade diferente da influência de j' em j.

Se, por um lado, o sucesso do auxílio à decisão depende crucialmente do modo como o conjunto de critérios é definido (Rogers et al., 2000, p. 6), por outro, nessa proposição, o modo como o relacionamento intercritérios é mensurado e expressado também é crucial.

- Forte complementação (entre 0,6 e 1,0) - quando o critério se relaciona muito pouco, tem escassos temas em comum, e não há nenhuma dependência;

- Complementação moderada (entre 0,4 e 0,6, inclusos) - quando os critérios têm relacionamento mediano, existindo alguns temas em comum;

- Pouca complementação (entre 0,1 e 0,4) - quando os critérios têm grande relacionamento, vários temas em comum e há dependência entre eles.

0 método proposto não se ocupa em definir os meios de mensurar esses relacionamentos, mas apenas de como eles são incorporados ao modelo. A escala utilizada tem incremento monotônico em 0,1 , variando de 0,1 até 1 .

Abordagem semelhante para resolver o problema da interação entre critérios é proposta para a família de métodos ELECTRE e PROMETHEE, por meio de uma ponderação que modela três possibilidades de interação entre os critérios (Corrente et al., 2012, p. 473; Figueira et al., 2009, p. 483).

\subsection{Definição da matriz linha $W d a$ importância dos critérios}

É sabido que os critérios não têm sempre a mesma importância, então é útil definir um peso $\left(\mathrm{w}_{\mathrm{j}}\right)$ associado a cada critério j. A distribuição de pesos aos critérios de modo a representar a sua importância relativa é sempre um ponto delicado (Rogers et al., 2000, p. 83). Tais pesos representam a força ou a importância desse critério (Marichal, 1999, p. 6).

A importância dos critérios é definida na matriz linha $W_{j}$, que contém os pesos agregados a cada critério, sendo esses definidos para dar perfil à escolha desejada. Caso não se deseje definir nenhum perfil, todos os valores $w_{j}$ recebem o valor 1 (um), que é fator neutro em um produto.

0 vetor peso tem uma importância considerável dentro dessa proposta, pois ele interfere diretamente na escolha das alternativas. Os valores atribuídos para a formação do vetor peso têm a função de ponderar a escolha para algum ponto, dando perfil às alternativas desejadas.

\subsection{Definição do tau de corte}

$0 \operatorname{tau}(\tau)$ de corte é uma contribuição da abordagem descritiva da tomada de decisão que, ao invés de trabalhar com a maximização de resultados, afirma que uma solução eleita pode ser somente "suficientemente satisfatória" (Façanha \& Yu, 2011, p. 61), ou seja, uma otimização precisa não é necessária, sendo suficiente estar próximo a essa otimização (Deming, 1990, p. XX), admitindo elementos de limitação da racionalidade do decisor e balanceando o modelo (Tietz, 1992, p. 299, 303).

Sua adoção também atende ao requisito da falta de precisão de julgamento típica das ciências de engenharia (Roy \& Vanderpooten, 1996, p. 24), em que se procura, pela incorporação de elementos de subjetividade, compensar essa falta adotando-se um limiar que, se alcançado, é dito suficiente (computa-se o valor um), e se não alcançado, é dito insuficiente (computa-se o valor zero).

0 círculo virtuoso da teoria da decisão apresenta que boas decisões são tomadas quando o modelo decisório incorpora tanto elementos de uma abordagem prescritiva, ou seja, como as decisões devem ser tomadas, embasadas na lógica, e elementos da abordagem descritiva, que relata como as decisões são tomadas, com grande carga de subjetividade e não explicadas por meio de modelos lógicos (Façanha $\& \mathrm{Yu}, 2011$, p. 52). Essa teoria é corroborada em experimentos em ambas abordagens (Reis \& Löbler, 2012, p. 414)

Por fim, é possível afirmar que o bom convívio entre a intuição e a lógica pode ser alcançado pelo emprego, quando possível, de métodos matemáticos (Kaufmann, 1975, p. 34).

Observa-se, também, como vantagem de seu uso, a descaracterização do modelo proposto como compensatório, pela soma aditiva, quando o bom desempenho em um item compensa o mau desempenho em outro. Assim, garante-se a vantagem dos modelos não compensatórios para a proposição do MEM.

Pelo modelo do MEM, a determinação de números entre 0 (zero) e 1 (um) adotados nas sucessivas multiplicações em seus mecanismos internos deve ser cuidadosa e não figurar próxima a 1. É interessante experimentar valores de modo que a solução supere o tau em aproximadamente $80 \%$ dos casos.

0 valor de $80 \%$ justifica-se no princípio de Pareto, o qual afirma que em qualquer população que contribui para um mesmo efeito haverá muitos triviais e poucos vitais (Juran, 1998, p. 5.20-21; Seleme \& 
Stadler, 2008, p. 88). A análise de Pareto (em que é aplicado o princípio homônimo) é amplamente utilizada, com sucesso (Juran \& Godfrey, 1998, p. AV.8; Juran, 1998, p. 5.24).

Cabe destacar inclusive que o valor de $80 \%$ é apenas um direcionador para indicar os itens vitais, podendo esse valor figurar nas dezenas vizinhas (Seleme \& Stadler, 2008, p. 88).

\subsection{Cálculo da matriz $B$}

A matriz B é a nova matriz de pagamentos, que traz as combinações das alternativas, tomadas $\mathrm{n}$ a $\mathrm{n}$ em suas linhas, e a combinação dos critérios, tomados dois a dois e multiplicados entre si.

Para o alcance da matriz B, são adotados os seguintes procedimentos:

- Normalização da matriz de pagamento original;

- Determinação do novo conjunto de alternativas e critérios;

- Cálculo dos elementos $b_{1 m}$ da matriz B.

A seguir são expandidos os procedimentos acima listados para alcance da matriz B.

\subsubsection{Normalização da matriz de pagamento original}

A normalização é útil quando se fazem medições em diferentes escalas, diferentes unidades, e facilita a manipulação das avaliações (Romero, 1996, p. 25-26). É utilizada a Equação 2, por sua capacidade de definir todos os critérios em um sentido de preferência crescente e de normalizar os valores, comparando-os com a amplitude da medição, o que é mais preciso do que fazê-lo por um valor máximo ou mínimo.

Equação 2. Normalização da matriz de pagamentos (Santos, 2011, p. 208).

$$
a_{i j}^{\prime}=\frac{\left|a_{i j}-\operatorname{pior}\left(a_{j}\right)\right|}{\left|\operatorname{melhor}\left(a_{j}\right)-\operatorname{pior}\left(a_{j}\right)\right|}, \forall i, j
$$

A essa matriz a normalizada dá-se a denominação $A_{i, j}^{\prime}$; ela é determinada conforme a Equação 2, onde $\mathrm{a}_{\mathrm{ij}}$ é o item $\mathrm{a}_{\mathrm{ij}}$ normalizado, considerando as melhores e piores avaliações, por critério, e, por isto, o sentido de preferência desses. A matriz A', assim, contém todas as avaliações, na mesma escala, e com o mesmo sentido de preferência.

\subsubsection{Determinação do novo conjunto de alternativas e de critérios}

Considerando $\mathrm{n}$ como sendo o número de escolhas que devem ser feitas, o novo conjunto de alternativas é determinado como ilustrado na Equação 3:
Equação 3. Determinação da quantidade de alternativas por combinação matemática.

$l=C_{n}^{i}=\frac{i !}{n !(i-n) !}$

onde 1 é o índice das alternativas da matriz B, cuja quantidade é determinada pela combinação das i alternativas, tomadas $n$ a $n$, sem repetição.

De modo análogo à determinação do novo conjunto de alternativas, também são determinados o novo conjunto de critérios, como ilustrado na Equação 4:

Equação 4. Determinação da quantidade critérios por combinação matemática.

$m=C_{n}^{j}=\frac{j !}{n !(j-n) !}$

onde $\mathrm{m}$ é o índice de critérios da matriz $\mathrm{B}$, cuja quantidade é determinada pela combinação dos $\mathrm{j}$ critérios, tomados $\mathrm{n}$ a $\mathrm{n}$, sem repetição.

\subsubsection{Cálculo dos elementos $b_{l m}$ da matriz $B$}

Para o cálculo da matriz B é necessária uma primeira matriz B' que conterá os elementos $b^{\prime}{ }_{\mathrm{jm}}$, que são os elementos $b_{\mathrm{jm}}$ antes da aplicação do tau de corte.

Os elementos b' ${ }_{j m}$ da matriz B' são calculados a partir de uma funçẫo de média ponderada simples, onde, para todo item l, tomam-se as avaliações contidas na $\mathrm{a}_{\mathrm{ij}}$ da matriz A' junto com os pesos contidos no vetor $\mathrm{W}$.

Assim sendo, admitindo-se que todo item 1 representa um vetor conjunto $\mathrm{L}$, que contém os índices i da matriz A' utilizados para sua determinação na combinação das alternativas, e de modo análogo, todo item $m$ representa um vetor $M$ que contém os índices j da matriz A' utilizados para sua determinação na combinação dos critérios, é proposta a Equação 5, que agrega a essa matriz B' também os elementos da matriz $\mathrm{C}$ de complementaridade entre os critérios.

Equação 5. Determinação dos elementos da matriz B'.

$b^{\prime}{ }_{l m}=\frac{\sum_{i} \sum_{j} a_{i j}^{\prime}}{n^{2}} \cdot \frac{\sum_{j} w_{j}}{n} \cdot c^{\prime}, \forall i \in L, \forall j \in M$

onde a' são os elementos da matriz A', $w_{\mathrm{j}}$, os elementos do vetor de pesos W e c' é a média dos índices de complementaridade entre critérios recuperados a partir dos elementos da matriz $\mathrm{C}$ de complementaridade entre critérios.

Para $\mathrm{n}$ igual a dois $(\mathrm{n}=2)$, ou seja, quando se deseja somente escolher duas alternativas concomitantemente, basta utilizar os valores presentes na matriz $\mathrm{C}$ de 
complementaridade entre critérios, mas para o caso de $n$ maior do que dois $(n>2)$, ou seja, deseja-se escolher mais do que duas alternativas concomitantemente, tem-se a necessidade de determinar um novo c', que abranja todas as complementaridades entre critérios.

Para tal, é calculado esse índice considerando-se todos os critérios envolvidos, paritariamente, e calculada a média aritmética simples de suas parcelas. 0 número total de itens, e, decorrente disso, também o de parcelas, é dado pelo denominador da Equação 6, que ilustra o cálculo de c' utilizado na Equação 5.

Equação 6. Cálculo do índice de complementaridade entre critérios.

$c^{\prime}=\frac{\sum c_{i j^{\prime}}}{n \times(n-1)}, \forall j, j^{\prime} \in M$

onde $c_{\mathrm{ij}}$ é o relacionamento de complementaridade entre critérios presente na matriz $\mathrm{C}$, sendo que os índices $\mathrm{j}$ e $\mathrm{j}$ ' variam de acordo com o número de alternativas a serem escolhidas, e, combinadas entre si, pertencem ao conjunto $\mathrm{M}$ que contém os índices dos critérios envolvidos na equação.

Por fim, o tau de corte é aplicado, conforme ilustrado na Equação 7, e onde é determinada a matriz B.

Equação 7. Aplicação do tau de corte.

$b_{l m}=\left\{\begin{array}{l}1 \rightarrow b^{\prime}{ }_{l m} \geq \tau \\ 0 \rightarrow b^{\prime}{ }_{l m}<\tau\end{array}\right.$

Assim, o maior valor obtido pela Equação 8 entre todas as alternativas combinadas indica a melhor (ou melhores) alternativa(s), onde o número de alternativas varia de acordo com o valor de corte $(\tau)$ aplicada no método.

Equação 8. Função aditiva para determinação do índice para escolha do melhor conjunto de alternativas.

$$
\frac{\sum_{m=1}^{k} b_{l m}}{k}, \forall l
$$

\section{Aplicação numérica}

\subsection{Definição do problema}

0 problema adotado para ilustração prática do método é o de recrutamento, no qual procura-se escolher três entre dez candidatos para vagas de emprego e no qual o desempenho acadêmico do histórico escolar dos candidatos nas disciplinas profissionalizantes é considerado como critério. Na Tabela 1 é apresentado o conjunto de critérios adotados para o problema de escolha.
Tabela 1. Legenda das disciplinas (critérios).

\begin{tabular}{ll}
\hline & \multicolumn{1}{c}{ Critério } \\
\hline 1 & Inovação Tecnológica \\
2 & Planejamento Estratégico Industrial \\
3 & Gerência da Manutenção \\
4 & Arranjo Físico e Localização \\
5 & Logística \\
6 & Administração de Recursos Humanos \\
7 & Administração da Produção \\
8 & Ciências Ambientais \\
9 & Gestão Ambiental \\
10 & Organização Industrial \\
11 & Custos da Produção \\
12 & Engenharia Econômica \\
13 & Gestão da Qualidade Total \\
14 & Controle Estatístico da Qualidade \\
15 & Gerência de Projetos \\
16 & Análise de Projetos Industriais \\
17 & Planejamento e Controle da Produção \\
18 & Ergonomia e Segurança do Trabalho \\
\hline
\end{tabular}

Assim, como é uma escolha de três candidatos entre dez, à luz de 18 disciplinas, a matriz B terá 120 alternativas ( 10 tomados 3 a 3), à luz de 816 critérios (18 tomados 3 a 3).

\subsection{Matriz de pagamentos}

Os dados que compõem essa matriz de pagamentos (ou julgamentos) foram extraídos aleatoriamente de dez egressos do curso de bacharel em Engenharia de Produção de uma instituição de ensino superior.

A Tabela 2 mostra o desempenho de dez candidatos (alternativas), nomeados de A1 a A10, em cada uma das 18 disciplinas (critérios) definidas na Tabela 1.

\subsection{Definição da matriz de complementaridade entre critérios}

A elaboração dessa matriz tem por intuito conflitar as disciplinas específicas do curso de Engenharia de Produção, com o propósito da obtenção do grau de relacionamento, de acordo com sua escala de influência. Quanto menor o valor, mais as disciplinas se parecem e menos se complementam, e quanto maior o valor, mais as disciplinas são distintas entre si, e, por isso, complementares.

Os dados obtidos na Tabela 3 foram coletados junto ao corpo docente do curso que serviu como objeto de estudo para ilustração do modelo proposto, por meio de um questionário eletrônico submetido via correio eletrônico a todos titulares das disciplinas em questão. 
Tabela 2. Matriz de pagamentos.

\begin{tabular}{ccccccccccccccccccc}
\hline & Cr. 1 & Cr. 2 & Cr. 3 & Cr. 4 & Cr. 5 & Cr. 6 & Cr. 7 & Cr. 8 & Cr. 9 & Cr. 10 & Cr. 11 & Cr. 12 & Cr. 13 & Cr. 14 & Cr. 15 & Cr. 16 & Cr. 17 & Cr. 18 \\
\hline A1 & 8,8 & 7,5 & 9,0 & 8,6 & 9,5 & 8,0 & 9,0 & 8,2 & 9,2 & 8,0 & 8,0 & 10 & 8,5 & 8,0 & 8,6 & 8,6 & 7,3 & 7,2 \\
A2 & 8,2 & 7,5 & 8,7 & 8,8 & 8,3 & 7,6 & 9,2 & 9,4 & 8,2 & 9,0 & 8,3 & 8,9 & 7,9 & 8,0 & 9,0 & 7,5 & 8,9 & 7,4 \\
A3 & 8,6 & 8 & 8,4 & 8,2 & 8,9 & 7,6 & 8,8 & 7,8 & 8 & 8,2 & 8,5 & 8,8 & 8,2 & 8,5 & 9,0 & 9,6 & 8,5 & 8,0 \\
A4 & 8,0 & 9,2 & 7,9 & 6,6 & 7,6 & 7,4 & 8,0 & 7,7 & 7,4 & 8,0 & 8,0 & 9,5 & 9,2 & 9,0 & 7,7 & 7,2 & 6,0 & 6,5 \\
A5 & 8,9 & 8,0 & 8,0 & 8,5 & 8,1 & 7,6 & 8,2 & 8,2 & 9,3 & 7,6 & 9,4 & 8,0 & 9,3 & 9,3 & 8,5 & 8,1 & 8,0 & 9,5 \\
A6 & 8,0 & 7,7 & 8,5 & 6,5 & 9,8 & 8,0 & 7,2 & 6,6 & 6,9 & 7,9 & 7,2 & 7,0 & 8,0 & 7,0 & 6,2 & 8,3 & 8,2 & 7,6 \\
A7 & 9,4 & 6,6 & 9,0 & 9,4 & 8,0 & 8,8 & 8,8 & 6,9 & 9,6 & 9,3 & 7,1 & 8,5 & 9,3 & 7,2 & 8,6 & 8,6 & 8,6 & 8,5 \\
A8 & 9,4 & 8,0 & 9,3 & 8,0 & 8,1 & 7,6 & 9,8 & 8,2 & 9,3 & 7,6 & 9,5 & 8,0 & 9,3 & 9,3 & 8,5 & 8,1 & 7,0 & 9,5 \\
A9 & 6,0 & 6,6 & 7,6 & 7,6 & 6,4 & 6,8 & 6,5 & 9,6 & 8,6 & 10 & 8,5 & 9,0 & 7,2 & 8,1 & 6,5 & 6,2 & 6,4 & 7,6 \\
A10 & 6,0 & 7,5 & 8,5 & 9 & 9,5 & 6,2 & 7,8 & 6,8 & 6,2 & 8,2 & 6,0 & 9,8 & 7,0 & 7,0 & 8,5 & 7,0 & 6,6 & 7,7 \\
\hline
\end{tabular}

Tabela 3. Matriz de complementaridade entre disciplinas.

\begin{tabular}{ccccccccccccccccccc}
\hline Cr. & $\mathbf{1}$ & $\mathbf{2}$ & $\mathbf{3}$ & $\mathbf{4}$ & $\mathbf{5}$ & $\mathbf{6}$ & $\mathbf{7}$ & $\mathbf{8}$ & $\mathbf{9}$ & $\mathbf{1 0}$ & $\mathbf{1 1}$ & $\mathbf{1 2}$ & $\mathbf{1 3}$ & $\mathbf{1 4}$ & $\mathbf{1 5}$ & $\mathbf{1 6}$ & $\mathbf{1 7}$ & $\mathbf{1 8}$ \\
\hline $\mathbf{1}$ & - & 0,5 & 0,8 & 0,4 & 0,5 & 0,9 & 0,2 & 1,0 & 1,0 & 0,3 & 1,0 & 0,9 & 0,5 & 0,8 & 0,5 & 0,9 & 0,5 & 0,5 \\
2 & 0,5 & - & 0,5 & 0,4 & 0,3 & 0,8 & 0,2 & 0,9 & 0,9 & 0,5 & 0,9 & 0,9 & 0,3 & 0,8 & 0,4 & 0,9 & 0,7 & 0,9 \\
3 & 0,8 & 0,5 & - & 0,4 & 0,5 & 0,9 & 0,5 & 0,9 & 0,9 & 0,5 & 1,0 & 1,0 & 0,8 & 1,0 & 0,6 & 1,0 & 0,5 & 0,9 \\
4 & 0,4 & 0,4 & 0,4 & - & 0,2 & 0,8 & 0,5 & 0,9 & 0,9 & 0,5 & 0,9 & 1,0 & 0,8 & 1,0 & 0,5 & 0,9 & 1,0 & 1,0 \\
5 & 0,5 & 0,3 & 0,5 & 0,2 & - & 0,6 & 0,5 & 0,9 & 0,9 & 0,5 & 0,7 & 0,9 & 0,5 & 0,6 & 0,4 & 0,8 & 0,8 & 0,8 \\
6 & 0,9 & 0,8 & 0,9 & 0,8 & 0,6 & - & 0,7 & 0,8 & 0,8 & 0,9 & 1,0 & 1,0 & 0,5 & 1,0 & 0,6 & 0,9 & 0,9 & 0,9 \\
7 & 0,2 & 0,2 & 0,5 & 0,5 & 0,5 & 0,7 & - & 0,6 & 0,5 & 0,5 & 0,7 & 0,9 & 0,4 & 0,6 & 0,2 & 0,9 & 0,7 & 0,7 \\
8 & 1,0 & 0,9 & 0,9 & 0,9 & 0,9 & 0,8 & 0,6 & - & 0,1 & 0,5 & 0,9 & 1,0 & 0,8 & 0,9 & 0,8 & 1,0 & 0,5 & 0,7 \\
9 & 1,0 & 0,9 & 0,9 & 0,9 & 0,9 & 0,8 & 0,5 & 0,1 & - & 0,5 & 0,9 & 1,0 & 0,8 & 0,9 & 0,8 & 1,0 & 0,5 & 0,7 \\
10 & 0,3 & 0,5 & 0,5 & 0,5 & 0,5 & 0,9 & 0,5 & 0,5 & 0,5 & - & 0,5 & 0,9 & 0,5 & 0,9 & 0,6 & 0,9 & 0,5 & 0,6 \\
11 & 1,0 & 0,9 & 1,0 & 0,9 & 0,7 & 1,0 & 0,7 & 0,9 & 0,9 & 0,5 & - & 0,3 & 0,8 & 0,4 & 0,9 & 0,3 & 0,5 & 0,8 \\
12 & 0,9 & 0,9 & 1,0 & 1,0 & 0,9 & 1,0 & 0,9 & 1,0 & 1,0 & 0,9 & 0,3 & - & 0,6 & 0,4 & 0,8 & 0,2 & 0,5 & 0,9 \\
13 & 0,5 & 0,3 & 0,8 & 0,8 & 0,5 & 0,5 & 0,4 & 0,8 & 0,8 & 0,5 & 0,8 & 0,6 & - & 0,5 & 0,5 & 0,7 & 0,7 & 0,8 \\
14 & 0,8 & 0,8 & 1,0 & 1,0 & 0,6 & 1,0 & 0,6 & 0,9 & 0,9 & 0,9 & 0,4 & 0,4 & 0,5 & - & 0,8 & 0,5 & 0,5 & 0,7 \\
15 & 0,5 & 0,4 & 0,6 & 0,5 & 0,4 & 0,6 & 0,2 & 0,8 & 0,8 & 0,6 & 0,9 & 0,8 & 0,5 & 0,8 & - & 0,3 & 0,8 & 0,7 \\
16 & 0,9 & 0,9 & 1,0 & 0,9 & 0,8 & 0,9 & 0,9 & 1,0 & 1,0 & 0,9 & 0,3 & 0,2 & 0,7 & 0,5 & 0,3 & - & 0,5 & 0,9 \\
17 & 0,5 & 0,7 & 0,5 & 1,0 & 0,8 & 0,9 & 0,7 & 0,5 & 0,5 & 0,5 & 0,5 & 0,5 & 0,7 & 0,5 & 0,8 & 0,5 & - & 0,5 \\
18 & 0,5 & 0,9 & 0,9 & 1,0 & 0,8 & 0,9 & 0,7 & 0,7 & 0,7 & 0,6 & 0,8 & 0,9 & 0,8 & 0,7 & 0,7 & 0,9 & 0,5 & - \\
\hline
\end{tabular}

Assim, quanto mais as disciplinas forem divergentes, mais elas complementam o perfil de uma escolha múltipla e essa situação é beneficiada no modelo proposto, pois para a empresa contratante não interessam candidatos com o mesmo perfil e sim com perfis diferentes que se completam. Para exemplificar, em termos práticos, não é interessante ter dois especialistas em Gestão Ambiental e nenhum em Planejamento e Controle da Produção, sendo preferivel ter um especialista em cada área.

É mister notar que a matriz $\mathrm{C}$ de complementaridade (ou influência) entre critérios é uma matriz quadrada, espelhada na sua diagonal principal, e que sua diagonal principal não possui valores, pois não é possível haver relacionamento da disciplina com ela própria.

\subsubsection{Definição da importância dos critérios}

A importância dos critérios é definida na matriz linha $W_{j}$, que contém os pesos agregados a cada critério, sendo esses definidos para dar perfil à escolha desejada. Caso não se deseje definir nenhum perfil, todos os valores $w_{j}$ recebem o valor 1 .

Os valores devem estar no intervalo entre 0 e 1 , e variar de acordo com cada critério, conforme visualizado na Tabela 4. Os valores foram escolhidos aleatoriamente por função própria em planilha eletrônica.

0 vetor peso tem uma importância considerável dentro dessa proposta, pois interfere diretamente na escolha das alternativas. Os valores atribuídos para a formação do vetor peso têm a função de ponderar a escolha para algum ponto, dando perfil às alternativas desejadas.

\section{Resultados}

Nesta seção são apresentados os resultados para as opções de escolha tripla, ou seja, para a escolha de três alternativas concomitantemente.

Antes disto, apresenta-se na Tabela 5 o resultado ordenado por média ponderada. 
Tabela 4. Matriz vetor peso.

\begin{tabular}{ccccccccccccccccccc}
\hline Critério & $\mathbf{1}$ & $\mathbf{2}$ & $\mathbf{3}$ & $\mathbf{4}$ & $\mathbf{5}$ & $\mathbf{6}$ & $\mathbf{7}$ & $\mathbf{8}$ & $\mathbf{9}$ & $\mathbf{1 0}$ & $\mathbf{1 1}$ & $\mathbf{1 2}$ & $\mathbf{1 3}$ & $\mathbf{1 4}$ & $\mathbf{1 5}$ & $\mathbf{1 6}$ & $\mathbf{1 7}$ & $\mathbf{1 8}$ \\
\hline Peso & 0,8 & 0,9 & 0,2 & 0,5 & 1,0 & 0,3 & 0,7 & 0,2 & 0,3 & 0,7 & 0,3 & 1,0 & 0,9 & 0,7 & 0,5 & 0,4 & 0,9 & 1,0 \\
\hline
\end{tabular}

Tabela 5. Resultado da ordenação por média ponderada.

\begin{tabular}{ccc}
\hline Ordenação & Alt. & Média \\
\hline $1 .{ }^{\circ}$ & A8 & 8,58 \\
$2{ }^{\circ}$ & A5 & 8,47 \\
$3 .^{\circ}$ & A7 & 8,46 \\
$4 .^{\circ}$ & A1 & 8,44 \\
$5{ }^{\circ}$ & A3 & 8,42 \\
$6 .{ }^{\circ}$ & A2 & 8,38 \\
$7 .^{\circ}$ & A4 & 7,83 \\
$8{ }^{\circ}$ & A6 & 7,59 \\
$9 .{ }^{\circ}$ & A10 & 7,52 \\
10. & A9 & 7,51 \\
\hline
\end{tabular}

Para a definição do tau de corte, considera-se que o modelo é resultado do produto de três fatores (Equação 5: avaliações da alternativa à luz do critério; pesos dos critérios; e índice de complementaridade). Admitindo-se um desempenho satisfatório de 50\% $(0,5)$ para as alternativas à luz dos critérios, a média dos pesos $\overline{\mathrm{w}}=0,628$ e a média dos índices de complementaridade $\overline{\bar{c}}=0,693$, resulta-se em um índice de 0,217. Admite-se um tau de corte para avaliar o desempenho como suficiente em 0,2, e, para a análise de sensibilidade, varia-se o tau de corte para 0,21 e 0,19 .

0 valor do tau de corte arbitrado em 0,2, por admitir esse como um desempenho satisfatório, corrobora a análise de sensibilidade, considerando-se os parâmetros em (a) porcentagem de alternativas escolhidas e (b) porcentagem da melhor alternativa, ilustrados na Figura 1.

Na Figura 1a observa-se que próximo ao valor de 0,2 , para qualquer tipo de escolha, atende-se ao critério da primeira escolha estar próxima a $80 \%$, e em 1 b observa-se que também próximo a 0,2 o método retorna poucas alternativas tidas como as melhores, característica discriminatória desejável em métodos de escolha.

Para a escolha tripla em estudo neste trabalho, observa-se na Figura 2 o comportamento ideal do método próximo ao tau igual a 0,2 , na qual a primeira alternativa escolhida figura próxima a $80 \%$, e na região do gráfico próximo a essa o método retorna uma ou duas alternativas para serem escolhidas.

\subsection{Para a escolha de três alternativas}

Ainda considerando a Tabela 5, para escolher três alternativas em conjunto é intuitivo optar pelo conjunto $\{A 5 ; A 7 ; A 8\}$, opções que figuram nas três primeiras posições. Contudo, considerando o mesmo problema proposto para a ilustração de uma escolha tripla, ou seja, a mesma matriz de pagamentos, o mesmo vetor de pesos dos critérios, a mesma matriz de critérios complementares e o mesmo valor do tau de corte, propõe-se uma outra solução pela utilização do MEM.

A combinação de dez alternativas tomadas em grupos de três resulta em um conjunto de 120 combinações diferentes. Por isto, prefere-se apresentar somente as 20 primeiras e as 20 últimas.

Na Tabela 6 apresenta-se o resultado obtido pela utilização do MEM para selecionar três alternativas. A melhor opção é a combinação obtida com \{A2; A7; A8\}, que ocupam as posições iniciais $1,3 \mathrm{e}$ 6 (não respectivamente). Demonstrando-se assim, novamente, que o melhor conjunto de três opções não é necessariamente o conjunto das três primeiras opções.

Os resultados da aplicação do tau de corte da alternativa $\{A 2, A 7, A 8\}$ eleita pelo MEM como melhor conjunto de três alternativas concomitantes é apresentado na Figura 3. Os pontos pretos em cima da linha 0,2 indicam as ocasiões que 0 valor resultante da Equação 5 superam o tau de corte, e os pontos pretos em cima do eixo das abscissas indicam as ocasiões em que isso não ocorre. São 816 ocasiões para o problema abordado. Esse gráfico ilustra o efeito não compensatório do método, pois os picos do gráfico não compensam os vales e toda vez que tau é superado, pontua da mesma forma.

Verifica-se no gráfico da Figura 3 que há poucos pontos no eixo das abscissas e muitos no eixo do tau de corte (80,8\% dos pontos), ilustrando o motivo da eleição da alternativa $\{A 2 ; A 7 ; A 8\}$ como o melhor conjunto de três alternativas possíveis, dentro do conjunto de alternativas iniciais.

Comparam-se os resultados da melhor escolha apontada pelo MEM com os da escolha intuitiva (os três melhores posicionados na ordenação por média ponderada) e a alternativa de pior desempenho, apresentados graficamente na Figura 4. Opta-se por ilustrar apenas os 100 últimos desempenhos dos 816 para melhor ilustrar o desempenho conjunto das alternativas, uma vez que o gráfico contendo as comparações e os 816 desempenhos seria confuso em demasia. É observado na Figura 4 que a alternativa $\{A 6 ; A 9 ; A 10\}$, no trecho apresentado, tem um 

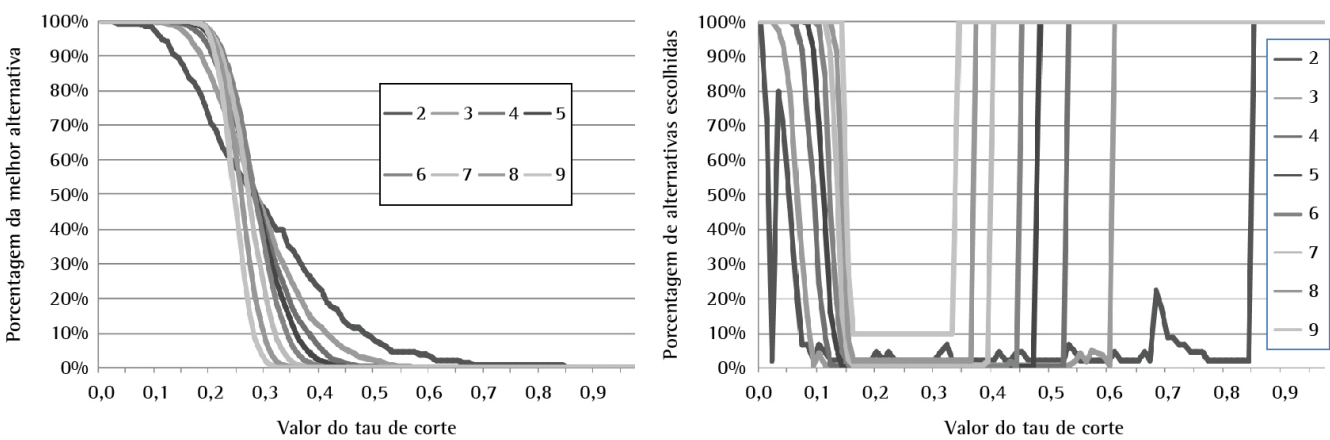

Figura 1. Análise de sensibilidade do tau de corte para (a) índice de porcentagem da melhor alternativa e (b) porcentagem de alternativas escolhidas em primeiro lugar.

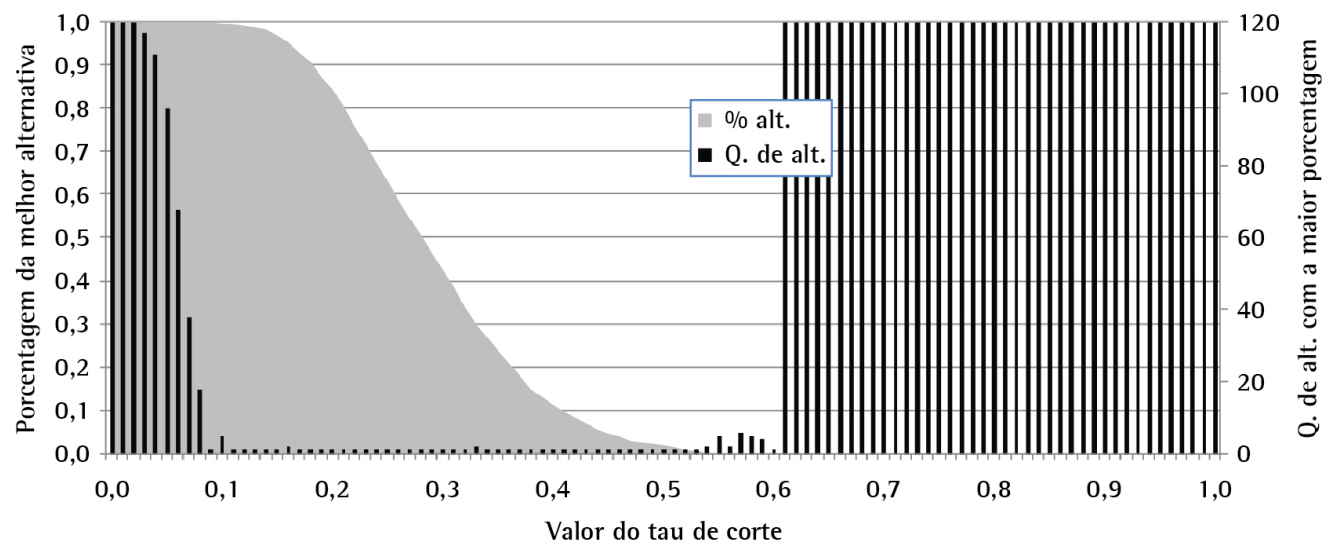

Figura 2. Análise de sensibilidade do tau de corte para o problema de escolha tripla.

Tabela 6. Resultado da ordenação para três escolhas $(\tau=0,20)$.

\begin{tabular}{cccccccc}
\hline \multicolumn{2}{c}{20 primeiras posições } & & \multicolumn{3}{c}{ 20 últimas posições } \\
\cline { 1 - 3 } \cline { 6 - 7 } Ord. & Alt. & \% & & Ord. & Alt. & $\%$ \\
\hline 1 & Alt. 2-7-8 & 84,6 & & 101 & Alt. 6-7-10 & 41,7 \\
2 & Alt. 1-2-8 & 83,0 & & 102 & Alt. 6-7-9 & 41,3 \\
3 & Alt. 1-7-8 & 82,5 & & 103 & Alt. 5-6-9 & 41,2 \\
4 & Alt. 3-7-8 & 82,4 & & 104 & Alt. 5-6-10 & 41,2 \\
5 & Alt. 2-3-8 & 82,4 & & 105 & Alt. 8-9-10 & 40,3 \\
6 & Alt. 1-3-8 & 81,6 & & 106 & Alt. 3-6-9 & 39,5 \\
7 & Alt. 2-3-7 & 81,5 & & 107 & Alt. 3-6-10 & 39,3 \\
8 & Alt. 1-2-7 & 81,5 & & 108 & Alt. 5-9-10 & 38,5 \\
9 & Alt. 1-2-5 & 81,4 & & 109 & Alt. 1-6-10 & 37,7 \\
10 & Alt. 2-5-7 & 81,1 & & 110 & Alt. 2-6-10 & 36,4 \\
11 & Alt. 1-2-3 & 80,8 & & 111 & Alt. 2-6-9 & 36,2 \\
12 & Alt. 5-7-8 & 80,6 & & 112 & Alt. 1-6-9 & 36,2 \\
13 & Alt. 1-3-7 & 80,5 & & 113 & Alt. 7-9-10 & 34,2 \\
14 & Alt. 2-5-8 & 80,4 & & 114 & Alt. 3-9-10 & 32,5 \\
15 & Alt. 3-5-7 & 80,3 & & 115 & Alt. 2-9-10 & 31,5 \\
16 & Alt. 2-3-5 & 80,1 & & 116 & Alt. 1-9-10 & 30,0 \\
17 & Alt. 1-5-7 & 80,0 & & 117 & Alt. 4-6-10 & 25,2 \\
18 & Alt. 1-5-8 & 79,9 & & 118 & Alt. 4-6-9 & 25,1 \\
19 & Alt. 1-3-5 & 79,9 & & 119 & Alt. 4-9-10 & 20,1 \\
20 & Alt. 3-5-8 & 78,8 & & 120 & Alt. 6-9-10 & 15,2 \\
\hline
\end{tabular}

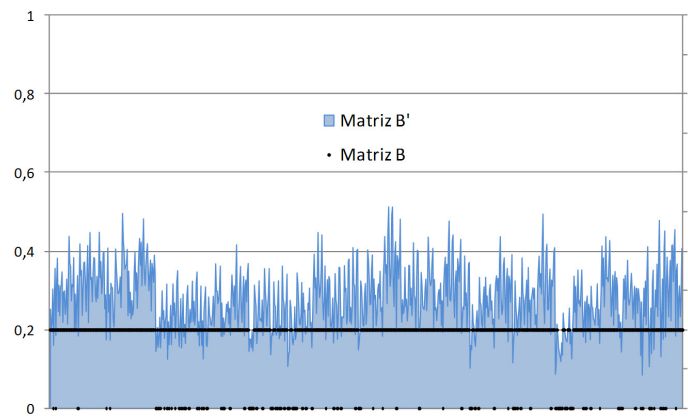

Figura 3. Gráfico ilustrativo da aplicação do tau de corte da alternativa $\{A 2, A 7, A 8\}$.

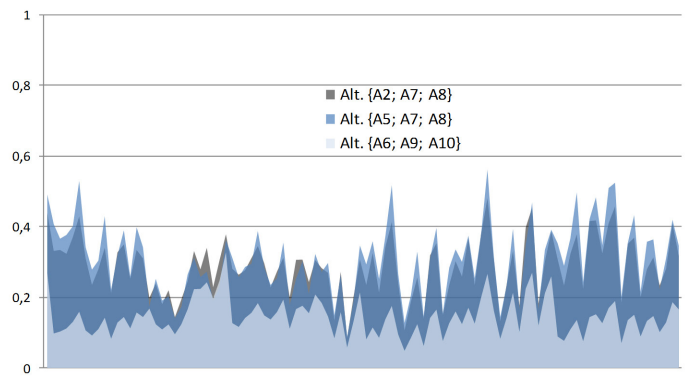

Figura 4. Comparação gráfica da melhor alternativa apontada pelo MEM, melhor alternativa intuitiva, e pior alternativa. 
desempenho aquém das demais, já que em poucas ocasiões supera o valor do tau de corte. Já a alternativa $\{A 5 ; A 7 ; A 8\}$ apresenta um bom desempenho, superando em diversos pontos o desempenho da alternativa $\{A 2 ; A 7 ; A 8\}$. Essa superação, contudo, não é compensatória, pois apesar de ter um desempenho superior nas 816 avaliações, não é na maioria das ocasiões que isso acontece, ilustrando novamente o efeito não compensatório do modelo, pelo qual não basta ter bons desempenhos individuais mas sim desempenhos mínimos coletivos.

No gráfico da Figura 4 verificam-se diversos picos isolados de bom desempenho, mas que não são suficientes para elevar a posição do conjunto, uma vez que a grande massa de dados não supera o tau de corte.

\subsection{Análise de sensibilidade}

A análise de sensibilidade em métodos multicritério discretos, com conjuntos finitos, busca observar como se dá a mudança da melhor alternativa quando os valores dos parâmetros (critérios) são alterados (Tanino, 1999, p. 7.25), ou, ainda, quando se estabelece um resultado, indaga-se quão próximo estão os resultados imediatamente inferiores (Kaufmann, 1975, p. 99).

É preocupação do processo de tomada de decisão garantir a estabilidade do sistema decisório (Saaty, 1991, p. 5). A análise de sensibilidade, em outra definição, ocupa-se em examinar quão robusta a ordenação de alternativas ou a escolha de uma única alternativa são em relação a pequenas mudanças nos componentes da MCDA (i.e., a estruturação do problema decisório, os valores dos critérios, os pesos dos critérios e as regras de decisão) (Malczewski, 2010, p. 382).

Continuando a análise de sensibilidade, foi aplicado o teste de correlação rho de Spearman, cujo resultado aponta uma alta correlação entre a ordenação proposta com o tau de 0,20 e as duas variações, sendo a correlação da primeira de 0,996 e, da segunda, 0,995, ambas em um nível que demonstra a robustez do resultado.

Por fim, analisa-se a oportunidade de uma escolha única, com vistas a uma escolha tripla, evidenciando a alternativa com maior potencialidade de complementaridade nesse tipo de problema $(n=3)$.

A alternativa $A 5$, que figura na segunda colocação na ordenação por média ponderada, em uma eventual escolha de três alternativas concomitantes não se caracteriza como uma "boa companheira" para os demais, sendo a alternativa mais adequada pela complementaridade a A2, que figura somente na sexta posição da ordenação por média ponderada, como ilustrado na Tabela 7.
Tabela 7. Frequência das alternativas entre as dez primeiras escolhas $(n=3)$.

\begin{tabular}{ccccc}
\hline & \multicolumn{3}{c}{ Frequência } & $\Sigma$ \\
\cline { 2 - 4 } Alt. & $\tau=0,20$ & $\tau=0,21$ & $\tau=0,19$ & \\
\hline A2 & 7 & 6 & 7 & 20 \\
A8 & 6 & 8 & 6 & 20 \\
A7 & 6 & 6 & 5 & 17 \\
A1 & 5 & 4 & 6 & 15 \\
A3 & 4 & 3 & 5 & 12 \\
A5 & 2 & 3 & 1 & 6 \\
\hline
\end{tabular}

\section{Conclusões}

\subsection{Quanto aos objetivos}

Este artigo ocupou-se em desenvolver um método multicritério que contemplasse a problemática de escolha múltipla, levando em consideração a complementaridade entre os critérios.

Esse objetivo é alcançado por meio da proposição do MEM, que adota nos seus procedimentos internos a matriz $\mathrm{C}$ de complementaridade entre critérios, incorporando a interação entre critérios ao modelo decisório, minimizando as interseções e maximizando as uniões das alternativas via critérios complementares.

\subsection{Quanto ao trabalho realizado}

Esta proposta, dentro da problemática de decisões, se encaixa melhor na problemática de portfólio (Belton \& Stewart, 2002, p. 15), uma vez que apresenta um subconjunto do conjunto original de alternativa(s) tida(s) como melhor(es) escolha(s) dentro das combinações possiveis e desenvolve uma abordagem prescritiva (Façanha \& Yu, 2011, p. 52) com elementos de subjeção da abordagem descritiva, mas diferencia-se pela possibilidade de determinar um número conhecido previamente de alternativas.

Enfim, o MEM apresenta mecanismos internos que privilegiam o bom desempenho médio da coletividade, preterindo excelentes desempenhos individuais agregados a desempenhos não tão bons.

Adotou-se como hipótese nula (básica) da pesquisa a situação em que não se aceita a priori que os $\mathrm{n}$ elementos de uma ordenação compõem o melhor grupo de $\mathrm{n}$ elementos por não considerar a sinergia intracritérios. Essa hipótese se confirma no experimento que simula uma escolha tripla, pois o melhor conjunto de três alternativas não é o conjunto das três melhores alternativas.

0 resultado do presente trabalho enriquece a área de conhecimento da engenharia de decisão com o desenvolvimento de um método multicritério para 
resolução de um problema ainda não tratado que evidencia um novo nicho acadêmico a ser explorado.

A limitação dos métodos multicritério, que apesar de tratarem com coerência e lógica os dados coletados afirmam que o resultado alcançado é válido somente para aquela pessoa ou grupo de pessoas (ou fornecedores dos dados) originário dos dados (Rogers et al., 2000, p. 189), ainda é válida para a proposta deste trabalho. Assim sendo, uma limitação não exclusiva do MEM, mas ainda existente nele, é a validade do resultado ter a mesma abrangência da fonte dos dados.

\section{Referências}

Abramczuk, A. A. (2009). A prática da tomada de decisão. São Paulo: Atlas.

Almeida, A. T., \& Duarte, M. D. 0. (2011). A multi-criteria decision model for selecting project portfolio with consideration being given to a new concept for synergies. Pesquisa Operacional, 31(2), 301-318. http://dx.doi. org/10.1590/S0101-74382011000200006

Almeida-Dias, J., Figueira, J. R., \& Roy, B. (2010). Electre Tri-C: A multiple criteria sorting method based on characteristic reference actions. European Journal of Operational Research, 204(3), 565-580. http://dx.doi. org/10.1016/j.ejor.2009.10.018

Bana e Costa, C. A. (1993). Três convicções fundamentais na prática do apoio à decisão. Pesquisa Operacional, 13(1), 9-20.

Belton, V., \& Stewart, T. J. (2002). Multiple Criteria Decision Analysis: An Integrated Approach. London: Springer. http://dx.doi.org/10.1007/978-1-4615-1495-4

Bhushan, N., \& Rai, K. (2004). Strategic decision making?: applying the analytic hierarchy process. London; New York: Springer.

Brans, J. P., Vincke, P., \& Mareschal, B. (1986). How to select and how to rank projects: The Promethee method. European Journal of Operational Research, 24(2), 228238. http://dx.doi.org/10.1016/0377-2217(86)90044-5

Carmines, E. G., \& Zeller, R. A. (1979). Reliability and validity assessment. Beverly Hills: Sage Publications.

Corrente, S., Figueira, J. R., \& Greco, S. (2012). Interaction of Criteria and Robust Ordinal Regression in Bi-polar PROMETHEE Methods. In 14th International Conference on Information Processing and Management of Uncertainty in Knowledge-Based Systems, Catania.

Deming, W. E. (1990). Qualidade: a revolução da administração. Tradução Francis Henrik Aubert; Maria Esmene Comenale; Aurea Consenza Dal Bó. Rio de Janeiro: Marques-Saraiva.

Ehrgott, M. (2005). Multicriteria optimization (Vol. 491, 2nd ed.). New Zeland: Springer Verlag.

Façanha, S. L. De 0., \& Yu, A. S. O. (2011). Abordagem integrada. In A. S. 0. Yu (Ed.), Tomada de decisão nas organizações (pp. 51-75). São Paulo: Saraiva.

Figueira, J. R., Greco, S., \& Roy, B. (2009). ELECTRE methods with interaction between criteria: An extension of the concordance index. European Journal of Operational
Research, 199(2), 478-495. http://dx.doi.org/10.1016/j. ejor.2008.11.025

Figueira, J. R. M., Mousseau, V., \& Roy, B. (2005). ELECTRE methods. In J. R. M. Figueira, S. Greco \& M. Ehrgott (Eds.), Multiple Criteria Decision Analysis: State Of The Art Surveys (pp. 133-162). London: Kluwer Academic Publishers.

Goicoechea, A., Hansen, D. R., \& Duckstein, L. (1982). Multiobjective decision analysis with engineering and business applications. Michigan: Wiley. PMid:6179711.

Gomes, L. F. A. M. (2007). Teoria da decisão. São Paulo: Thomson.

Gomes, L. F. A. M., \& Gomes, C. F. S. (2012). Tomada de decisão gerencial: enfoque multicritério (4. ed.). São Paulo: Atlas.

Gomes, L. F. A. M., Gomes, C. F. S., \& Almeida, A. T. (2002). Tomada de decisão gerencial: enfoque multicritério. São Paulo: Atlas.

Gomes, L. F. A. M., González Araya, M. C., \& Carignano, C. (2004). Tomada de decisões em cenários complexos. São Paulo: Thomson.

Gomes, L. F. A. M., \& Lima, M. M. P. P. ( 1991). TODIM: Basics and application to multicriteria ranking of projects with environmental impacts. Foundations of Control Engineering, 16(3-4), 113-127.

Gravetter, F. J., \& Wallnau, L. B. (2008). Statistics for the Behavioral Sciences. Belmont: Cengage Learning.

Hansson, S. 0. (2005). Decision Theory: a brief introduction. Stockholm.

Juran, J. M. (1998). The quality improvment proccess. In: J. M. Juran, A. B. Godfrey (Eds.), Juran's quality handbook. (pp. 5.1-73, 5. ed.). New York: McGraw-Hill.

Juran, J. M., \& Godfrey, A. B. (1998). Juran's quality handbook. 5. ed. New York: McGraw-Hill. PMid:9494721.

Kaufmann, A. (1975). A ciência da tomada de decisão. Tradução Francisco José de Albuquerque Souza. Rio de Janeiro: Zahar.

Malczewski, J. (2010). Multiple criteria decision analysis and Geographic information systems. In M. Ehrgott, J. R. M. Figueira \& S. Greco (Eds.), Trends in Multiple Criteria Decision Analysis (pp. 369-396). London: Springer. http://dx.doi.org/10.1007/978-1-4419-5904-1_13

Marichal, J.-L. (1999). Aggregation operators for multicriteria decision aid (Ph.D. Thesis). University of Liège.

Marichal, J.-L., \& Roubens, M. (2000). Determination of weights of interacting criteria from a reference set. European Journal of Operational Research, 124(3), 641650. http://dx.doi.org/10.1016/S0377-2217(99)00182-4

Markowitz, H. (1952). Portfolio selection. The Journal of Finance, 7(1), 77-91.

Pagano, M., \& Gauvreau, K. (2004). Princípios de bioestatística. Tradução Sérgio de Castro Paiva. São Paulo: Thomson.

Raiffa, H. (2002). Decision Analysis: A Personal Account of How it Got Started and Evolved. Operations Research, 50(1), 179-185. http://dx.doi.org/10.1287/ opre.50.1.179.17797

Reis, E., \& Löbler, M. L. (2012). 0 processo decisório descrito pelo indivíduo e pepresentado nos sistemas de apoio à decisão. Revista de Administração 
Contemporânea, 16(3), 397-417. http://dx.doi. org/10.1590/S1415-65552012000300005

Rogers, M., Bruen, M., \& Maystre, L.-Y. (2000). Electre and decision support?: methods and applications in engineering and infrastructure investment. Boston: Kluwer Academic. http://dx.doi.org/10.1007/978-14757-5057-7

Romero, C. (1996). Análisis de las decisiones multicriterio. Madrid: lsdefe.

Roy, B., \& Vanderpooten, D. (1996). The European school of MCDA: Emergence, basic features and current works. Journal of Multi-Criteria Decision Analysis, 5(1), 2238. http://dx.doi.org/10.1002/(SICl) 10991360(199603)5:1<22::AID-MCDA93>3.0.CO;2-F

Saaty, T. L. (1991). Método de análise hierárquica. Tradução Wainer da Silveira e Silva. São Paulo: Makron.

Saaty, T. L., \& Vargas, L. G. (2006). Decision Making With the Analytic Network Process: Economic, Political, Social And Technological Applications With Benefits, Opportunities, Costs And Risks. New York: Springer.

Santos, J. R. (2011). Métodos de análise. In A. S. O. Yu (Ed.), Tomada de decisão nas organizações (pp. 189-222). São Paulo: Saraiva.

Seleme, R., \& Stadler, H. (2008). Controle de Qualidade: as ferramentas essenciais. São Paulo: Editora lbpex.

Tanino, T. (1999). Sensitive Analysis in MCDM. In T. Gál, T. J. Stewart \& T. Hanne (Eds.). Multicriteria Decision
Making: Advances in MCDM Models, Algorithms, Theory, and Applications (pp. 7.1-7.29). London: Springer. http://dx.doi.org/10.1007/978-1-4615-5025-9_7

Tietz, R. (1992). Semi-normative theories based on bounded rationality. Journal of Economic Psychology, 13(2), 297 314. http://dx.doi.org/10.1016/0167-4870(92)90035-6

Triantaphyllou, E. (2000). Multi-Criteria Decision Making Methods: A Comparative Study. Lousiana: Springer. PMid:10944406. http://dx.doi.org/10.1007/978-14757-3157-6

Vargas, L. G. (1990). An overview of the analytic hierarchy process and its applications. European Journal of Operational Research, 48(1), 2-8. http://dx.doi. org/10.1016/0377-2217(90)90056-H

Vincke, P. (1992). Multicriteria decision-aid. Tradução Marjorie Gassner. Chichester, England: Wiley.

\section{Agradecimentos}

Este estudo só foi possível pelo apoio financeiro da CAPES, a quem os autores gostariam de agradecer. Também registra-se agradecimento aos revisores do manuscrito, que apontaram sugestões precisas, melhoraram o texto e sua apresentação em diversos aspectos.

\title{
Proposal for a multicriteria method for multiple choice
}

\begin{abstract}
This research paper aims to propose a multicriteria solution to the multiple-choice problematic within the same decision problem, based on the hypothesis that the ' $n$ ' best alternatives in a ranking listing are not necessarily the best set of ' $n$ ' alternatives. Through experimental research, a recruiting problem where three candidates should be chosen from a set of ten is simulated. MEM - Multiplex Electionis Methodus - offers a solution to this problem starting from the traditional modeling of the decision problems, with a payment matrix and weights vector, and aggregating a matrix of complementarity among criteria. The results show that the best triple set is not the set of the three best ranked options in a simple ranking list, thus fulfilling the research paper's objective by confirming the stated hypothesis, thereby contradicting the intuitive decision based on the assumption that the ' $n$ ' first alternatives compose the best set of ' $n$ ' alternatives.
\end{abstract}

\section{Keywords}

Multiple-choice. Multicriteria method. Decision theory. 